\title{
Quantum-Chemical Calculations for the Electronic Absorption Spectra of Certain Anthocyanidins
}

\author{
V. I. Deineka ${ }^{a, *}$ and Ya. Yu. Kulchenko ${ }^{a}$ \\ ${ }^{a}$ Belgorod National Research State University, Belgorod, 308015 Russia \\ *e-mail:deineka@bsu.edu.ru
}

Received August 30, 2020; revised August 30, 2020; accepted September 10, 2020

\begin{abstract}
Semi-empirical PM3 calculations are made for the electronic absorption spectra of colored forms of nonmethylated anthocyanidins (aurantinidin, pelargonidin, cyanidin, and delphinidin). The position of the absorption band peak is shown to depend on the orientation of hydroxyl groups in the aglycone structure. The observed tendency toward a bathochromic shift after $\mathrm{OH}$ groups are added to the ring generally corresponds to experimental data. The results for the uncharged quinonoid forms of pelargonidin (a hypsochromic shift of absorption bands) do not in this case agree with the prevailing opinion of specialists about the bathochromic shift. While this opinion is confirmed by DFT calculations, there are still no reliable experimental data on their validity. The formation of single-charged (negatively) quinonoid structures is clearly the reason for the bathochromic shift of absorption bands, which is consistent with the experimental data. When the charge multiplicity increases (to -2), a hypsochromic shift of absorption bands to the experimentally observed yellow color of anthocyanins in alkaline solutions is again predicted.
\end{abstract}

Keywords: quantum-chemical calculations, PM3, configurational interaction, anthocyanins, simulation of electronic absorption spectra

DOI: $10.1134 / \mathrm{S} 0036024421070086$

\section{INTRODUCTION}

Anthocyanins are unique compounds of a vast class of flavonoids. Their distinguishing chemical characteristic is the existence of several $\mathrm{pH}$-dependent forms [1, 2]. Anthocyanins are glycosides of anthocyanidins. The flavylium form is responsible for the red color of fruits, flowers, leaves, and other parts of plants, with tints that depend on the structure of the anthocyanin. However, the variety of plant colors is governed not only by the flavylium form but by complex supramolecular structures that can include other forms of anthocyanins as well. It is known that when $\mathrm{pH}$ grow from 1 or less (a condition for the existence of anthocyanins and anthocyanidines only in flavylium I, Scheme 1), this form becomes a colorless pseudo-base due to nucleophilic attack by water molecules (hemiacetal II, Scheme 1). The hemiacetal form is in equilibrium with a weakly colored cis-chalcone form (III, Scheme 1) that can also isomerize into a weakly colored trans-chalcone form (IV, Scheme 1).

However, there is another possible course of the transformation of the flavylium form as $\mathrm{pH}$ rises (Scheme 2): deprotonation with the formation of uncharged (Va and Vb) quinonoid structures, the number of which depends on the structure of anthocy- anins (the existence of glycoside substituents at position 5 and sometimes at position 7). These structures can then transform into single (VI, Scheme 2) or (for anthocyanins without a glycoside substituent at position 5) double charged quinonoid structures. The quinonoid forms are considered to be colored too, but at wavelengths other than the flavylium form.

Though there are many works on transitions between the anthocyanin and anthocyanidin forms, their electronic spectra (except for that of flavylium) are unknown. The color of quinonoid forms is assumed to be blue shifted (with magenta coloring) relative to the flavylium form, while the negatively charged quinonoid form is blue [1-4]. Unfortunately, it is impossible to obtain the spectra of individual forms of natural anthocyanins (except for those of flavylium) because of the simultaneous presence of many forms in different ratios, and the instability of non-flavylium forms. In [5], we proposed a way of separating the spectra of anthocyanins by obtaining difference spectra. However, this approach has yet to be fully substantiated, though HPLC with spectra measured by a diode matrix detector eliminates the second reason: the instability of forms. It is therefore of great importance to calculate the electronic absorption spectra of different forms of anthocyanins and anthocyanidins via quantum chemistry. Several works on ab 
<smiles>[R3]O/C(=C\c1c(O)cc(O)cc1O[R3])C(=O)c1cc([R3])c(O)c([R])c1</smiles>

Scheme 1. Transformations of the flavylium form in the direction of hydration. Forms: I, flavylium; II, pseudo-base; III, cischalcone; IV, trans-chalcone.

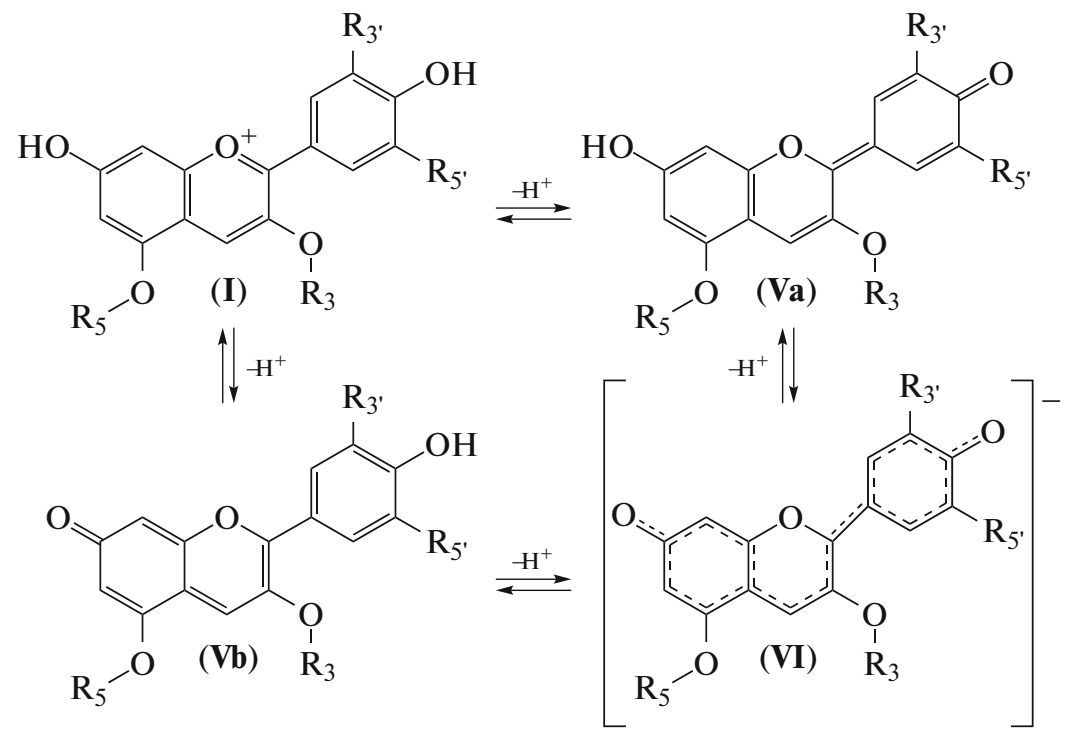

Scheme 2. Transformations of the flavylium form in the deprotonation direction. Forms: I, flavylium; Va and Vb, uncharged quinonoid; VI, charged quinonoid.

initio (including different means of density functional theory) and semi-empirical calculations of anthocyanins and anthocyanidins [6-11] have been published, with less attention given to non-flavylium forms [9, $11]$.

The aim of this work was to perform quantumchemical calculations of the electronic absorption spectra of some anthocyanidins in the flavylium and charged and uncharged quinonoid forms. Instead of anthocyanins, anthocyanidins were chosen as compounds that had the simplest structures but greater possibilities, due to the diversity of their forms.

\section{EXPERIMENTAL}

Semi-empirical quantum-chemical calculations were performed using the HyperChem 8.0 program package. The geometry was optimized in vacuo using the semi-empirical PM3 technique [12]. The PolakRibiere algorithm with a gradient limit of $0.0001 \mathrm{kcal} /(\AA \mathrm{mol})$ and a limited number of cycles (1000) was used to optimize the molecular geometry. The electronic spectra were calculated under the condition of single excitation, and only for singlet states at configurational interaction with an energy limit $(10 \mathrm{eV})$ in the PM3 and ZINDO/S approaches. 
In this work, we used experimental wavelengths obtained in methanol $+1 \% \mathrm{HCl}$ solvent reported in [7]: $499 \mathrm{~nm}$ for aurantinidin (3,4',5,6,7-tetrahydroxyflavylium); $520 \mathrm{~nm}$ for pelargonidin (3,4',5,7-tetrahydroxyflavylium); $535 \mathrm{~nm}$ for cyanidin $\left(3,3^{\prime}, 44^{\prime}, 5,7-\right.$ pentahydroxyflavylium); and $546 \mathrm{~nm}$ for delphinidin $\left(3,3^{\prime}, 4^{\prime}, 5,5^{\prime}, 7\right.$-hexahydroxyflavylium).

\section{RESULTS AND DISCUSSION \\ Calculations for Flavylium Ions}

In making our calculations, we found that the result $\left(\lambda_{\max }\right.$ value) depends on the orientation of hydroxyl groups in the structure of flavylium ions. In the above works, no attention was given to the orientation of these groups, except for the orientation of the $\mathrm{OH}$ group at position 3, in one of which the planarity of the molecule is violated [7]. The orientation of $\mathrm{OH}$ groups in the anthocyanidin ion was denoted by positive signs if the $\mathrm{OH}$ group was oriented clockwise, and by negative signs for groups with the opposite orientation (Scheme 3).

The total energies of particles, the wavelength of the electronic transition in the absorption spectrum, and dipole moments obtained for ions of pelargonidin (anthocyanidin), which has fewest hydroxyl groups of all the common natural anthocyanidins, are presented in Table 1.

The difference between the calculated wavelengths of the absorption band $\left(\lambda_{\max }\right)$ for different orientations of hydroxyl groups (except the $\mathrm{OH}$ group at position 3) was $\sim 9 \mathrm{~nm}(485.62-494.55 \mathrm{~nm})$. Here, the difference between conformation energies was no more than $3.84 \mathrm{kcal} / \mathrm{mol}$, which is comparable to the energy of the thermal motion of molecules. Average value $\lambda_{\max }=$ $489.29 \mathrm{~nm}$ may therefore be taken as a tentative parameter. Due to rapid (vertical) electronic transi-

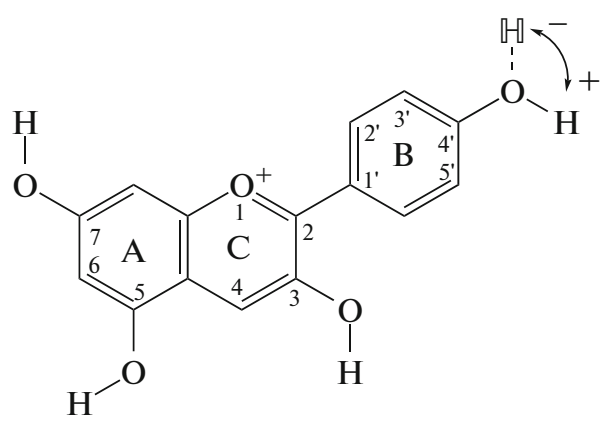

Scheme 3. Structure of the flavylium form of pelargonidin.

tions in different conformations, however, real spectra can be the sum of spectra of individual conformations, with weight determined by, e.g., conformation analysis. Bands in the spectra of anthocyanins or anthocyanidins can therefore be broadened or asymmetric even if there is only one allowed transition.

An analysis of the data in Table 1 shows that the orientation of the dipole moment at position 5 contributes greatly to the energy of state: $\lambda_{\max }=486.5 \pm$ $0.9 \mathrm{~nm}$ in the positive direction and $493.5 \pm 1.4 \mathrm{~nm}$ in the negative. Of the two calculation procedures (PM3 and ZINDO/S), the first yielded results closer to the experimental value $(520 \mathrm{~nm})$, so only this procedure was used below.

In our calculations, we excluded the counterclockwise orientations of the $\mathrm{OH}$ group at position 3 , since they create great steric hindrances in particles and violations in planarity. For a conformation with all negative directions of $\mathrm{OH}$ groups (a planar structure of all conformations with positive orientation of the $\mathrm{OH}$ group at position 3), the torsion angle of $\mathrm{O}_{1}-\mathrm{C}_{2}-\mathrm{C}_{1^{\prime}}$ $\mathrm{C}_{2}$ grows to $45.5^{\circ}$, but reduced conjugation between $\mathrm{B}$

Table 1. Results from quantum-chemical calculations for the flavylium form of pelargonidin ( $E$ is the particle energy, $\lambda$ is the transition wavelength, and $\mu$ is the particle dipole moment)

\begin{tabular}{c|c|c|c|c|c|c|c}
\hline \multicolumn{6}{c|}{ Orientations of OH groups at positions } & \multirow{2}{*}{$-E, \mathrm{kcal} / \mathrm{mol}$} & \multicolumn{2}{|c|}{$\lambda, \mathrm{nm}$} & \multirow{2}{*}{$\mu, \mathrm{D}$} \\
\cline { 1 - 6 }+7 & 5 & 3 & 4 & & PM3 & ZINDO/S & \\
\hline+ & + & + & + & 3412.4932 & 485.62 & 468.00 & 1.931 \\
+ & + & + & - & 3412.3516 & 486.46 & 468.24 & 1.921 \\
+ & - & + & + & 3409.6530 & 491.79 & 472.12 & 2.608 \\
- & + & + & + & 3412.4114 & 486.57 & 468.12 & 4.104 \\
+ & - & + & - & 3409.5432 & 492.65 & 472.35 & 0.984 \\
- & + & + & - & 3412.3263 & 487.38 & 468.36 & 3.587 \\
- & - & + & + & 3410.2277 & 493.69 & 472.27 & 0 \\
- & - & + & - & 3410.1749 & 494.55 & 472.50 & 1.398 \\
- & - & - & - & 3410.4853 & 483.22 & & 2.506 \\
- & + & - & - & 3412.3497 & 469.51 & & 4.907 \\
\hline
\end{tabular}


Table 2. Results from quantum-chemical calculations for cyanidin ions

\begin{tabular}{|c|c|c|c|c|c|c|c|}
\hline \multicolumn{5}{|c|}{ Orientations of $\mathrm{OH}$ groups at positions } & \multirow{2}{*}{$-E, \mathrm{kcal} / \mathrm{mol}$} & \multirow{2}{*}{$\lambda, \mathrm{nm}$} & \multirow{2}{*}{$\mu, \mathrm{D}$} \\
\hline 3 & 5 & 7 & $3^{\prime}$ & $4^{\prime}$ & & & \\
\hline & & & & & \multicolumn{3}{|c|}{ Type I conformation } \\
\hline+ & - & - & + & + & 3511.7828 & 499.16 & 4.78 \\
\hline+ & - & + & - & - & 3510.9769 & 502.92 & 1.193 \\
\hline+ & - & + & - & + & 3509.2081 & 504.68 & 2.766 \\
\hline \multirow[t]{2}{*}{+} & + & - & - & + & 3512.0558 & 497.57 & 6.185 \\
\hline & & & & & \multicolumn{3}{|c|}{ Type II conformation } \\
\hline+ & - & - & - & - & 3511.6523 & 504.81 & 2.272 \\
\hline+ & + & - & + & - & 3512.1126 & 503.40 & 5.994 \\
\hline+ & + & - & + & + & 3514.0668 & 499.80 & 6.112 \\
\hline \multirow[t]{2}{*}{+} & + & + & + & + & 3514.1656 & 499.14 & 3.929 \\
\hline & & & & & \multicolumn{3}{|c|}{ Type II conformation (3-) } \\
\hline- & - & - & + & + & 3511.9115 & 502.50 & 4.136 \\
\hline- & + & + & + & + & 3513.9567 & 485.10 & 4.37 \\
\hline
\end{tabular}

and $\mathrm{C}$ rings has virtually no effect on the position of $\lambda_{\max }$. The same torsion angle changes slightly (to $47^{\circ}$ ) for the positive orientation of the $\mathrm{OH}$ group at position 5 , and $\lambda_{\max }$ shifts hypsochromically by $\sim 14 \mathrm{~nm}$ (from $483.2^{\circ}$ to $469.5^{\circ}$ ).

We found no correlations between $\lambda_{\max }$ and the dipole moment for a particle in vacuo. However, it is entirely possible that this parameter could be important during the solvatochromic effects that occur upon moving from a vacuum to true polar solutions and expand the range of possible $\lambda_{\max }$ values for different particle conformations.

We did not perform calculations for all possible wavelengths, since the number of possible different orientations of hydroxide ions grows substantially upon moving to cyanidin (for which two types of conformations are possible, with $\mathrm{C}_{2}-\mathrm{O}_{1}-\mathrm{C}_{1}-\mathrm{C}_{2}$ torsion angles of $0^{\circ}$ for the first and $180^{\circ}$ for the second) and delphinidin (which contains six $\mathrm{OH}$ groups). Results from calculating some $\mathrm{OH}$ group orientations for the flavylium form of cyanidin are summarized in Table 2.

The $\lambda_{\max }$ dispersion was less than $6 \mathrm{~nm}$ for positive orientations of the $\mathrm{OH}$ group at position 3 (average $\lambda_{\max }=501.4 \mathrm{~nm}$ ), and the difference between ground state energies was less than $5 \mathrm{kcal} / \mathrm{mol}$. Our calculations thus predicted a $12 \mathrm{~nm}$ bathochromic shift of $\lambda_{\max }$ upon moving from pelargonidin to cyanidin, which is less than the experimentally determined difference in wavelengths $(15 \mathrm{~nm})$. As with pelargonidin, we found that the ring lay outside the plane of conjugation for the negative orientation of the $\mathrm{OH}$ group at position 3 , and the range of $\lambda_{\max }$ was extended for electronic transitions in different conformations. To support the idea that electronic absorption spectra can be superpositionings of the individual spectra of different conformations, let us consider the experimental absorption spectrum of cyanidin, measured in a detector cuvette during the separation of anthocyanidins via reverse-phase HPLC in mobile 10 vol \% formic acid and 14 vol \% acetonitrile phase (Fig. 1).

Figure 1 shows that the experimental spectrum of cyanidin is a complex superpositioning of several individual bands with only one allowed transition, according to the data from quantum-chemical calculations for each particle conformation. Note that there was no analysis of experimental absorption spectra in any of the above works, and our intricate structure could be a consequence of vertical electronic transitions for the set of existing conformations.

The spread of $\lambda_{\max }$ for delphinidin was $20 \mathrm{~nm}$ at an average value of $505.7 \mathrm{~nm}$ for eight randomly selected conformations. This preceded a bathochromic shift of the absorption band upon moving from cyanidin to delphinidin, in accordance with the experimental data $\left(\lambda_{\max }\right.$ variation from 535 to $546 \mathrm{~nm}$ ). The observed increase in $\lambda_{\max }$ correlates with a rise in the particle energy in the ground state. The same dependence was also found for cyanidin, but only when the results were grouped according to conformation (Fig. 2; the coordinate axes were changed to compare three dependences in one graph).

A transition from pelargonidin to aurantinidin, achieved by adding an OH group at position 6 with the clockwise orientation of all groups, resulted in a $9 \mathrm{~nm}$ hypsochromic shift of the absorption band, which corresponded to the direction of the change in $\lambda_{\max }$ in the experimental electronic spectra. 


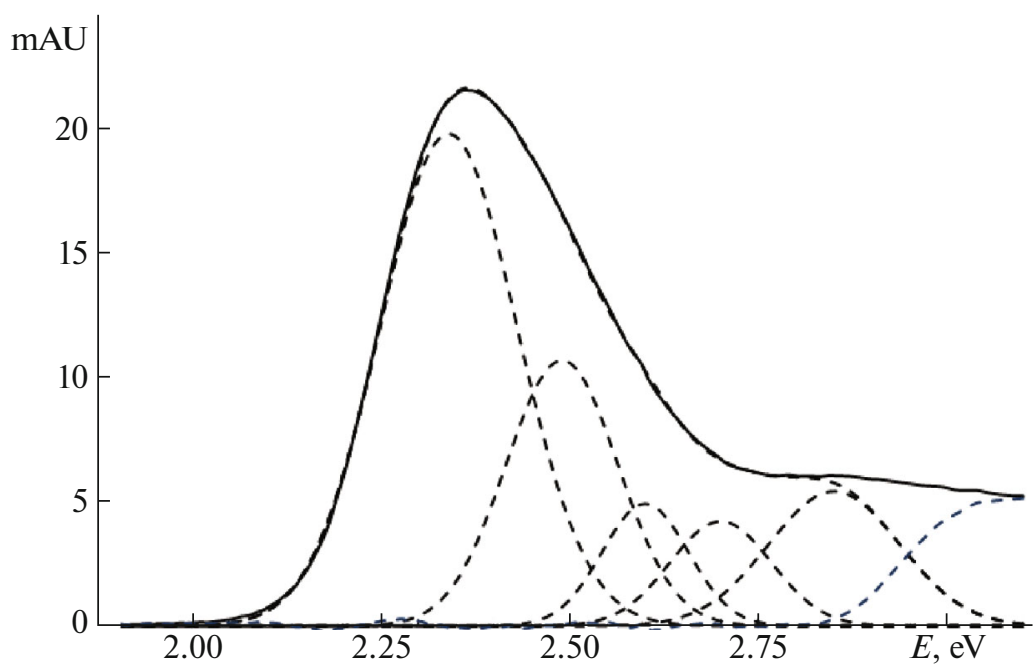

Fig. 1. Electronic absorption spectrum of cyanidin, fitted using individual Gaussian bands.

Unfortunately, our quantum-chemical calculations of changes in the electronic absorption spectra during the methylation of $\mathrm{OH}$ groups in the cyanidin (for peonidin) and delphinidin (for petunidin and malvidin) series were disappointing, due to the wide range of wavelengths found for different conformations of flavylium ions, with the experimental ones remaining unchanged in the first series and growing by only $1.0-1.5 \mathrm{~nm}$ in the second series.

\section{Calculations for Quinonoid Structures}

There are three possible structures for pelargonidin: 4'-, 5-, and 7, according to the keto group position (Scheme 4).

In our calculations, we excluded structures with the anticlockwise orientation of the hydroxyl group at position 3 caused by great steric hindrances that give the molecule a complex nonplanar conformation.

With the 4' quinonoid structure, the change in the orientation of $\mathrm{OH}$ groups at positions 5 and 7 weakly affects the wavelengths at the absorption maximum (Table 3); however, the average value of $405.3 \pm$ $0.7 \mathrm{~nm}$ does not agree with the view that a small bathochromic shift of the band wavelength occurs upon moving from flavylium to the quinonoid form $[9,11,13]$. For the most stable (in energy) 5-quinonoid form, the maximum shifts to $455.2 \pm 0.7 \mathrm{~nm}$, but this band is still shifted hypsochromically relative to the band of the flavylium form. Calculations in [9] regarding a number of synthetic anthocyanins with known spectral characteristics for flavylium and quinonoid structures showed that semi-empirical calculations of $\lambda_{\max }$ for quinonoid structures were less successful than ones made using DFT. The uncharged quinonoid form was not mentioned at all in [14], and the change in the color was attributed to the emergence of single- and double-charged quinonoid struc- tures. According to our data, a distinct shade of magenta appears only for anthocyanins acylated by substituted cinnamic acids, so this shift of the absorption band could be a consequence of intramolecular co-pigmentation [15].

The effect the position of the carbonyl group has on the planarity of the structure even with the positive orientation of the hydroxyl group at position 3 (Table 4) could be due to unexpected structural features of the quinonoid forms (relative to that of flavylium). Only the 4'-quinonoid form is planar in all conformations, and the conjugated bonds are out of plane for the 5- and 7-quinonoid structures. The change in the bond angle at carbon atom $2\left(\mathrm{O}_{1}-\mathrm{C}_{2}-\right.$

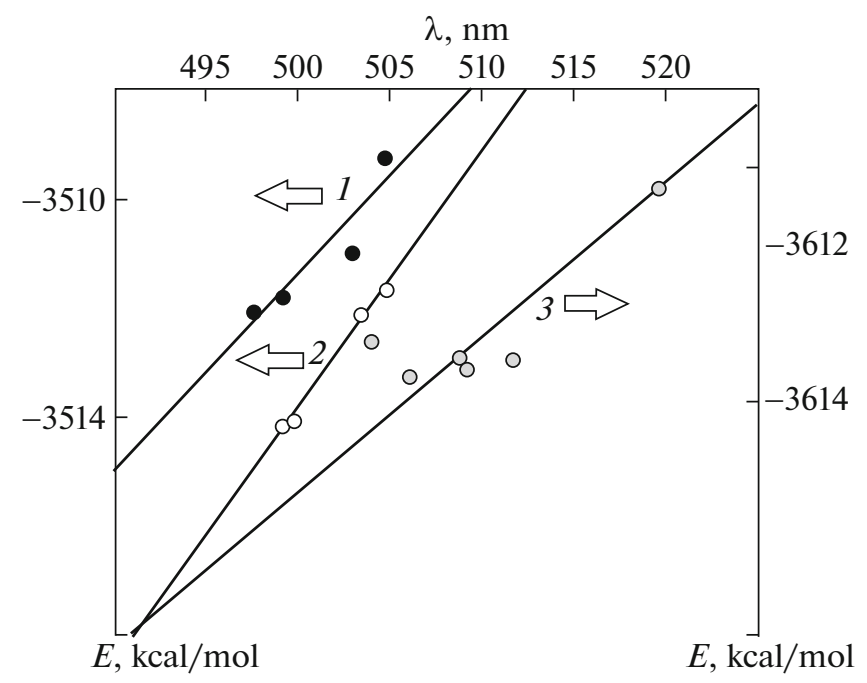

Fig. 2. Correlation between the calculated band maxima in the electronic absorption spectra and the calculated energies of ground state conformations of cyanidin: (1) type I conformation; (2) type II conformation; (3) delphinidin. 
<smiles>O=C1C=CC(=C2Oc3cc(O)cc(O)c3C=C2O)C=C1</smiles><smiles>O=c1cc(O)cc2oc(-c3ccc(O)cc3)c(O)cc1-2</smiles><smiles>O=c1cc2oc(-c3ccc(O)cc3)c(O)cc-2c(O)c1</smiles>

Scheme 4. Quinonoid structures of pelargonidin.

$\mathrm{C}_{1}$ ) testifies to the steric repulsion between rings $\mathrm{B}$ and $\mathrm{C}$. These are same reasons for the change in torsion angles: the angle of $\mathrm{O}_{1}-\mathrm{C}_{2}-\mathrm{C}_{1}-\mathrm{C}_{2}$, pushing ring $\mathrm{B}$ from the plane of conjugation along with rings $\mathrm{A}$ and $\mathrm{C}$, and the angle of $\mathrm{C}_{2}-\mathrm{C}_{3}-\mathrm{O}_{\mathrm{C} 3}-\mathrm{H}$ partially pushing the $\mathrm{OH}$ group out of the plane of the molecule. However, the weakening of the conjugation raises the wavelength of the absorption band maximum.

\section{Calculations for Charged Quinonoid Structures}

A specific feature of charged quinonoid structures with $\mathrm{a}-1$ charge is that there are two equivalent forms, e.g.,
- the 4'-quinonoid structure with deprotonation of the hydroxyl group at position 7;

- the 7-quinonoid structure with deprotonation of the hydroxyl group at position 4'.

Both structures are distorted notably, including the change in the orientation of the hydroxyl group at position 3. An appreciable bathochromic shift of the absorption maximum to $550-560 \mathrm{~nm}$ was also observed (Table 5). The blue color of aqueous anthocyanin solutions, observed experimentally in a number of cases when $\mathrm{pH}$ was increased, can provide the formation about this structure. Note that all of the calcu-

Table 3. Results from quantum-chemical calculations of uncharged quinonoid structures of pelargonidin

\begin{tabular}{|c|c|c|c|c|c|c|}
\hline \multirow{2}{*}{ Structure } & \multicolumn{4}{|c|}{ Orientation of $\mathrm{OH}$ groups at positions } & \multirow{2}{*}{$-E, \mathrm{kcal} / \mathrm{mol}$} & \multirow{2}{*}{$\lambda, \mathrm{nm}$} \\
\hline & 7 & 5 & 3 & 4 & & \\
\hline \multirow[t]{4}{*}{ 4'-Quinonoid } & + & + & + & & 3493.5349 & 405.42 \\
\hline & - & + & + & & 3492.9957 & 404.61 \\
\hline & + & - & + & & 3493.7887 & 405.86 \\
\hline & - & - & + & & 3493.8306 & 405.18 \\
\hline \multirow[t]{4}{*}{ 5-Quinonoid } & + & & + & + & 3497.2040 & 453.97 \\
\hline & + & & + & - & 3497.1413 & 454.00 \\
\hline & - & & + & + & 3498.1748 & 456.40 \\
\hline & - & & + & - & 3498.5490 & 456.35 \\
\hline \multirow[t]{4}{*}{ 7-Quinonoid } & & + & + & + & 3494.9837 & 450.20 \\
\hline & & + & + & - & 3495.0242 & 449.91 \\
\hline & & - & + & + & 3493.4099 & 447.47 \\
\hline & & - & + & - & 3468.0212 & 446.48 \\
\hline
\end{tabular}

Table 4. Effect of the carbonyl group's position on the planarity of quinonoid structures

\begin{tabular}{|c|c|c|c|c|c|c|}
\hline \multirow{2}{*}{ Structure } & \multicolumn{3}{|c|}{$\mathrm{OH}$ group orientation at position } & \multirow{2}{*}{$\begin{array}{l}\mathrm{O}_{1}-\mathrm{C}_{2}-\mathrm{C}_{1^{\prime}} \\
\text { bond angle }\end{array}$} & \multicolumn{2}{|c|}{ Torsion angle, deg } \\
\hline & 5 & 7 & $4^{\prime}$ & & $\mathrm{O}_{1}-\mathrm{C}_{2}-\mathrm{C}_{1^{\prime}}-\mathrm{C}_{2^{\prime}}$ & $\mathrm{C}_{2}-\mathrm{C}_{3}-\mathrm{O}_{\mathrm{C} 3}-\mathrm{H}$ \\
\hline \multirow[t]{2}{*}{$4^{\prime}$} & + & + & & 114 & 0 & 180 \\
\hline & - & - & & 114 & 0 & 180 \\
\hline \multirow[t]{2}{*}{5} & & + & + & 112 & -12.8 & -168.9 \\
\hline & & - & - & 112 & -12.5 & -168.7 \\
\hline \multirow[t]{2}{*}{7} & + & & + & 112 & -14.6 & -154.9 \\
\hline & - & & - & 112 & -15.9 & -132.2 \\
\hline
\end{tabular}


Table 5. Results from quantum-chemical calculations of pelargonidin quinonoid structures with a -1 charge

\begin{tabular}{|c|c|c|c|c|c|c|c|c|c|}
\hline \multirow{2}{*}{ Structure } & \multicolumn{4}{|c|}{ Orientation of $\mathrm{OH}$ groups at positions } & \multicolumn{3}{|c|}{$\begin{array}{l}\text { Angles in the ground states } \\
\text { of the structures }\end{array}$} & \multirow{2}{*}{$\begin{array}{c}-E \\
\mathrm{kcal} / \mathrm{mol}\end{array}$} & \multirow{2}{*}{$\lambda, \mathrm{nm}$} \\
\hline & 3 & 5 & 7 & $4^{\prime}$ & I* & II ${ }^{* *}$ & III** & & \\
\hline \multirow{2}{*}{$\begin{array}{l}4^{\prime}, \text { charge in } 7(-) \equiv 7, \\
\text { charge in } 4^{\prime}(-)\end{array}$} & + & - & \multirow{6}{*}{+} & & 112.4 & 20.7 & 14.4 & 3494.2075 & 560.45 \\
\hline & + & + & & & 112.4 & 21.0 & 14.8 & 3493.4878 & 555.98 \\
\hline \multirow{2}{*}{$\begin{array}{l}4^{\prime}, \text { charge in } 5(-) \equiv 5, \\
\text { charge in } 4^{\prime}(-)\end{array}$} & + & & & & 113.6 & -5.8 & -104.2 & 3495.2769 & 514.86 \\
\hline & + & & & & 113.7 & -5.7 & -103.8 & 3495.4416 & 516.05 \\
\hline \multirow{2}{*}{$\begin{array}{l}5, \text { charge in } 7(-) \equiv 7, \\
\text { charge in } 5(-)\end{array}$} & + & & & + & 112.1 & -7.2 & -177.8 & 3483.3197 & 434.87 \\
\hline & + & & & - & 112.0 & -6.6 & -178.1 & 3483.4473 & 434.45 \\
\hline
\end{tabular}

$* \mathrm{O}_{1}-\mathrm{C}_{2}-\mathrm{C}_{1}$, bond angle, ${ }^{* *} \mathrm{O}_{1}-\mathrm{C}_{2}-\mathrm{C}_{1}-\mathrm{C}_{2}$, torsion angle, and ${ }^{* * *} \mathrm{C}_{2}-\mathrm{C}_{3}-\mathrm{O}_{\mathrm{C} 3}-\mathrm{H}$ torsion angle, deg.

Table 6. Results from quantum-chemical calculations of pelargonidin quinonoid structures with a -2 charge (where $f$ is the oscillator strength)

\begin{tabular}{l|c|c|c|c|c|c}
\hline \multirow{2}{*}{ Structure } & \multicolumn{3}{|c|}{$\begin{array}{c}\text { Angles in the ground states } \\
\text { of the structures, deg }\end{array}$} & \multirow{2}{*}{$-E, \mathrm{kcal} / \mathrm{mol}$} & $\lambda, \mathrm{nm}$ & $f$ \\
\cline { 2 - 4 } & I & II & III & & & \\
\hline 4', charges in 7 and 5 5, & 112.3 & -40.3 & 7.6 & 3425.8875 & 457.06 & 0.191 \\
charges in 7 and 4' & & & & & 438.69 & 0.493 \\
7, charges in 5 and 4' & 113.2 & -54.0 & -3.7 & 3426.2969 & 457.01 & 0.100 \\
& & & & & 424.32 & 0.522 \\
\hline
\end{tabular}

lated wavelengths of absorption bands found in this work were hypsochromically shifted relative to the experimental data. This could be a consequence of the solvatochromic effect, especially since it was observed when water replaces methanol as a solvent (according to our data, there is a 10-20 nm hypsochromic shift after this substitution). The probability of similar structures existing among anthocyanins is high, since positions 7 and 4' usually remain free of substituents. The other two equivalent pairs of structures are observed more rarely in nature, since the $\mathrm{OH}$ group at position 5 is often glycosylated. However, a notable (though less pronounced) hypsochromic shift of absorption bands was obtained for these too (Table 5).

The double-charged anionic structures that unexpectedly separated into two partially equivalent structures (Table 6) were characterized by an abrupt drop in the absorption band wavelength to the yellow range of the electromagnetic spectrum, which is consistent with the experimentally observed changes in anthocyanins in alkaline media that are accompanied by the formation of green solutions (as a mixture of blue and yellow forms) and then gradually replaced by yellowcolored solutions even upon storage.

\section{REFERENCES}

1. P. Trouillas, J. C. Sancho-Garcia, V. de Freitas, et al., Chem. Rev. 116, 4937 (2016). https://doi.org/10.1021/acs.chemrev.5b00507

2. O. Dangles and J.-A. Fenger, Molecules 23, 1970 (2018). https://doi.org/10.3390/molecules23081970

3. J. C. Lima, C. Vautier-Giongo, A. Lopes, et al., J. Phys. Chem. A 106, 5851 (2002). https://doi.org/10.1021/jp014081c

4. P. M. Rose, V. Cantrill, M. Benohoud, et al., J. Agric. Food Chem. 66, 6790 (2018). https://doi.org/10.1021/acs.jafc.8b01044

5. V. I. Deineka, Ya. Yu. Kul'chenko, and L. A. Deineka, Russ. J. Phys. Chem. A 93, 572 (2019).

6. W. E. Kurtin and P.-S. Song, Tetrahedron 24, 2255 (1968). https://doi.org/10.1016/0040-4020(68)88127-X

7. K. Sakata, N. Saito, and T. Honda, Tetrahedron 62 , 3721 (2006).

https://doi.org/10.1016/j.tet.2006.01.081

8. E. H. Anouar, J. Gierschner, J.-L. Duroux, et al., Food Chem. 131, 79 (2012). https://doi.org/10.1016/j.foodchem.2011.08.034

9. A. A. Freitas, K. Shimizu, L. G. Dias, et al., J. Braz. Chem. Soc. 18, 1537 (2007). 
10. U. Terranova and D. R. Bowler, J. Chem. Theory Comput. 9, 3181 (2013). https://doi.org/10.1021/ct400356k

11. M. Rusishvili, L. Grisanti, S. Laporte, et al., Phys. Chem. Chem. Phys. 21, 8757 (2019). https://doi.org/10.1039/C9CP00747D

12. J. J. P. Stewart, J. Comput. Chem. 10, 209 (1989). https://doi.org/10.1002/jcc.540100208

13. R. Brouillard, S. Chassaing, G. Isorez, et al., in Recent Advances in Polyphenol Research, Ed. by C. Santos-
Buelga, M. T. Escribano-Bailon, and V. Lattanzio (Blackwell, London, 2010), Vol. 2, p. 1.

14. H. E. Khoo, A. Azlan, S. T. Tang, et al., Food Nutr. Res. 61, 1361779 (2017). https://doi.org/10.1080/16546628.2017.1361779

15. J. He, X. Li, G. T. M. Silva, F. H. Quina, et al., J. Braz. Chem. Soc. 30, 492 (2019). https://doi.org/10.21577/0103-5053.20180233

Translated by L. Chernikova 